selbst organisieren können. In der letzten Projektphase bis Februar 2009 wird deshalb eine Internetpräsentation erstellt, die Hinweise und Tipps zur Durchführung von Interviews geben soll. Dort werden Themen wie Interviewtechniken, Transkriptionsregeln, die erforderliche technische Ausrüstung, rechtliche Aspekte, Sicherung und Auswertung und vieles mehr erläutert. Dabei wird - ähnlich wie bei den eingangs erwähnten Seiten zu Archivfragen (www.FrauVerA.de) - mit Beispielen und
Links zu weiterführenden Informationen gearbeitet werden. Wir hoffen, damit Frauenvereinen und -verbänden ein Hilfsmittel an die Hand zu geben, das ihnen ermöglicht, selbst in dieser Form die Geschichte ihres Verbandes in „Erzählungen“ zu dokumentieren.

Selbstverständlich stehen wir auch gerne allen Interessierten für Auskünfte zur Verfügung (loeneke@addf-kassel.de oderwenzel@addf-kassel.de).

\title{
Deutsche Juristinnen von 1948 bis 1989
}

Nachdem Juristinnen während des Regimes des Nationalsozialismus weitgehend aus dem Berufsleben ausgeschlossen gewesen waren, kam ihnen nach 1945 der große Bedarf sowohl in der Anwaltschaft als auch in der Rechtsprechung zu Gute. War ihnen zuvor die Berufsausübung verwehrt worden, konnten Rechtsanwältinnen und Richterinnen nun davon profitieren, als nicht belastet zu gelten. Deutsche Juristinnen wurden mit Verteidigungen vor den Militärgerichten betraut, noch bevor die deutsche Justiz ihre Tätigkeit wieder aufgenommen hatte. Sie wirkten beim Aufbau der kommunalen Verwaltungen mit und wurden Richterinnen an Amts-, Land- und Oberlandesgerichten. Juristinnen fanden sich auch in den politischen Parteien. Viele teilten das Schicksal, ihren Ehepartner im Krieg verloren zu haben. Sie mussten nicht nur ihre Kinder allein erziehen, sondern auch für den Lebensunterhalt einer ganzen Familie sorgen.

Dr. Erna Scheffler, während des NS-Regimes vom Beruf ausgeschlossen, wurde im September 1951 als einzige Frau an das neu gegründete Bundesverfassungsgericht in Karlsruhe berufen. Dr. Gerda Krüger-Nieland und Elisabeth Krumme wurden Richterinnen der ersten Stunde am Bundesgerichtshof, Dr. Anne-Gudrun Meier-Scherling folgte einem Ruf an das Bundesarbeitsgericht, Dr. Maria Schwarz ging an das Bundessozialgericht und Charlotte Schmidt wurde Richterin am Bundesverwaltungsgericht.

Im August 1948 gründeten sieben Juristinnen ${ }^{1}$ in Dortmund wieder einen Verein für Juristinnen, nunmehr auch für Volkswirtinnen. Die „Vereinigung weiblicher Juristen und Volkswirte e.V.“ verstand sich als Nachfolgeorganisation des 1914 gegründeten „Deutschen Juristinnen-Vereins, e.V.“. Die 1. Vorsitzende Hildegard Gethmann aus Dortmund wollte weibliche Kräfte zur Wahrnehmung der Interessen von Frauen im öffentlichen Leben bündeln und verlangte dafür von jeder Frau, die dem Verein beitrat, die hohe Einsatzbereitschaft, die ihr selbst zu eigen war.

\section{Das Ringen um Artikel 3 Abs. 2 und 3 GG}

Die Verankerung der Gleichberechtigung von Frau und Mann in Artikel 3 des Grundgesetzes verlieh dem Anliegen der Frauen erstmals eine rechtliche Grundlage zur Durchsetzung der Gleichberechtigung in allen Rechtsgebieten. Bis es zur entsprechenden Fassung des Artikel 3 GG kam, hatte es im Parlamentarischen Rat einen zähen Machtkampf gegeben, dessen Ausgang im Wesentlichen dem Verhandlungsgeschick der Rechtsanwältin Dr. Elisabeth Selbert zu verdanken ist. Fortschrittlich hinsichtlich einer paritätischen Behandlung von Frau und Mann im Recht schon in ihrer Dissertation 1930, hielt sie für selbstverständlich, dass die Gleichberechtigung im Grundgesetz festgehalten wurde. Als eine von vier Frauen sah sie sich im Parlamentarischen Rat jedoch 61 männlichen Abgeordneten gegenüber, die es indiskutabel fanden, Frauen durch die Verfassung gleiche Rechte im Hinblick auf alle Rechtsgebiete zu gewähren. Dem stetigen Drängen Elisabeth Selberts wurde erst nachgegeben, als Frauen aus der gesamten Republik mit Protestschreiben von den Abgeordneten die volle Gleichberechtigung forderten. Infolgedessen legten Artikel 3 Absatz 2 und Absatz 3 des Grundgesetzes das Recht der Frau auf Gleichberechtigung und ein Verbot der Ungleichbehandlung von Frau und Mann fest. Alle hiermit unvereinbaren Gesetze, so das Grundgesetz weiter, sollten mit Ablauf des 31. Dezember 1953 keine Gültigkeit mehr haben. Das Erarbeiten von Vorschlägen für neue, mit der Verfassung vereinbare Gesetze war

\footnotetext{
1 Rechtsanwältin Hildegard Gethmann, Assessorin Luise Purps, Rechtsanwältin Dr. Ruth Rogalski-Rohwedder, Regierungsrätin Dr. Anna Schlieper, Anwaltsassessorin Alma Schmidt-Perchner, Anwaltsassessorin Annette Schücking und Rechtsanwältin Elisabeth Späth-Uden.
}

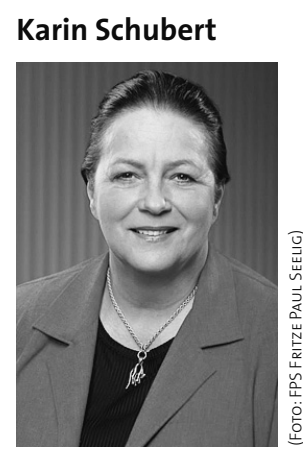

Senatorin a.D., Justizministerin a.D., Rechtsanwältin, Berlin 
die Kernarbeit der Vereinigung weiblicher Juristen und Volkswirte in den ersten Jahren nach ihrer Gründung.

\section{Ehe- und Familienrecht}

Ein besonderes Anliegen der Juristinnen war die Reform des Ehe- und Familienrechts. Unter maßgeblichem Einfluss der Rechtsanwältin Dr. Elisabeth Schwarzhaupt wurde zunächst mit dem Gleichberechtigungsgesetz von 1957 der so genannte „Stichentscheid“ - das Letztentscheidungsrecht - des Ehemannes abgeschafft. Erhalten blieb allerdings zunächst noch der „Stichentscheid“ des Vaters. Diesen erklärte das Bundesverfassungsgericht auf die Verfassungsbeschwerde von vier Müttern - unter ihnen die Rechtsanwältin Dr. h.c. Maria Müller-Lütgenau - 1959 für nichtig.

\section{Erst nachdem die damaligen djb-Vorsitzenden Dr. Lore Maria Peschel-Gutzeit und Dr. Gisela Wild den Alterna- tiventwurf auf einer Pressekonferenz präsentiert hat- ten, wurde Dr. Peschel-Gutzeit als Sachverständige im Rechtsausschuss des Bundestags angehört.}

Mit Dr. Elisabeth Schwarzhaupt - von 1961 bis 1966 Bundesgesundheitsministerin - als Vorsitzende des Sonderausschusses zur Reform des Nichtehelichenrechts wurde darüber hinaus nach langem Ringen die Stellung des nichtehelichen Kindes verbessert. Der Juristinnenbund - so hieß die Vereinigung weiblicher Juristen und Volkswirte seit 1959 - hatte 1966 unter der Ägide der Rechtsanwältin und Notarin Maria Kramer-Schulz zu einem Referentenentwurf, der die Rechte des nichtehelichen Kindes betraf, Stellung genommen, weil es ihn für verfassungswidrig und unzureichend hielt. Grund hierfür war insbesondere, dass der Entwurf zugrunde legte, dass das Wohl des Kindes vorbehaltlich eines Gegenbeweises am besten durch das Jugendamt als Beistand und nicht durch die Mutter gewährleistet sei. Das Gesetzgebungsverfahren schleppte sich immens; wesentlich auf das Drängen der damaligen 1. Vorsitzenden des Juristinnenbundes Dr. Renate Lenz-Fuchs ist es zurückzuführen, dass das Gesetz überhaupt noch in der laufenden Legislaturperiode verabschiedet werden konnte. Mit dem Gesetz über die Rechtsstellung der nichtehelichen Kinder vom 19. August 1969 wurde der Mutter das volle Sorgerecht zugesprochen; das nichteheliche Kind erhielt ein Erbrecht gegenüber dem Vater und Unterhaltsansprüche wie ein eheliches Kind. Auch wenn die Beistandschaft nicht gänzlich aufgehoben war, wurde sie immerhin durch eine gesetzliche Pflegschaft ersetzt.
Fast zeitgleich erfolgte die Debatte zur Reform des Ehe- und Scheidungsrechts. Eine von der Bundesregierung geforderte Kommission war 1968 mit der entsprechenden Vorbereitung beauftragt worden; der Juristinnenbund war durch die Oberlandesgerichtsrätin Dr. Anneliese Cuny vertreten. Sie erarbeitete Stellungnahmen zu zwei Gesetzentwürfen; wesentliche Aspekte dieser Stellungnahmen sind in das 1. Eherechtsreformgesetz von 1977 eingegangen. Kernpunkte waren das Zerrüttungsprinzip anstelle des Verschuldensprinzips bei der Ehescheidung sowie die grundsätzliche Eigenverantwortlichkeit der Partner nach der Scheidung neben einer Mitverantwortung gegenüber dem geschiedenen Ehegatten auf der Grundlage von fest umrissenen Unterhaltstatbeständen.

Die familienrechtlichen Bestrebungen des Juristinnenbundes sind untrennbar mit dem Namen Dr. Lore Maria Peschel-Gutzeit verbunden. 1975 - damals noch Familienrichterin am Hanseatischen OLG, später dreimalig Justizsenatorin - saß sie einer neu gegründeten Familienrechtskommission des Juristinnenbundes vor, deren Ziel es war, zu Entwürfen der Bundesregierung zur Neuregelung des Rechts der elterlichen Sorge Stellung zu nehmen. Die Kommission hielt die Regierungsvorschläge überwiegend für ungeeignet und entwickelte einen Alternativentwurf. Dieser enthielt unter anderem erstmalig ein generelles Verbot von Gewalt in der Familie sowie eigene Vorschläge zum Recht der elterlichen Sorge und dem Umgangsrecht nach Scheidung oder Verlust eines Elternteils. Der Vorschlag wurde der Öffentlichkeit im Jahr 1977 präsentiert, der Deutsche Bundestag wollte ihn jedoch zunächst ignorieren. Erst nachdem die damaligen djb-Vorsitzenden $^{2}$ Dr. Lore Maria Peschel-Gutzeit und Dr. Gisela Wild den Alternativentwurf auf einer Pressekonferenz präsentiert hatten, wurde Dr. PeschelGutzeit als Sachverständige im Rechtsausschuss des Bundestags angehört. Der Erfolg war groß: Teile des Alternativentwurfs sind wörtlich, andere Teile sinngemäß in das 1979 verabschiedete Gesetz zur Neuregelung der elterlichen Sorge eingegangen.

Neben zahlreichen anderen gesetzgeberischen und politischen Entwicklungen wie beispielsweise dem Unterhaltsänderungsgesetz von 1984 hat Dr. Lore Maria Peschel-Gutzeit als Vorsitzende der Familienrechtskommission des djb - gemeinsam mit der damaligen 1. Vorsitzenden des djb Renate Damm - auch maßgeblich die Entscheidung des Bundesverfassungsgerichts zur Ehelichkeitsanfech-

2 Seit 1977 hieß der vorherige „Juristinnenbund“ „Deutscher Juristinnenbund“ und wurde „DJB“ abgekürzt. Mit Einführung der Website www.djb.de wurde die Abkürzung in Kleinbuchstaben immer geläufiger. 
tung im Januar 1989 beeinflusst. Die Stellungnahme des djb in der mündlichen Verhandlung beinhaltete, dass ein uneingeschränktes Anfechtungsrecht des Kindes aus dem allgemeinen Persönlichkeitsrecht resultiere. Diese Haltung widersprach den Auffassungen der Bundesregierung sowie fast aller geladenen Verbände und Interessenvertretungen, dennoch ist ihr das Bundesverfassungsgericht weitgehend gefolgt.

Im Juni 1988 rief der djb den Gesetzgeber zu einer grundsätzlichen gesetzlichen Regelung der nichtehelichen Lebensgemeinschaft auf. Insbesondere waren hier die Bereiche Unterhalt, Auseinandersetzung bei Trennung, Kindeswohl sowie Erbund Steuerrecht angesprochen. Auf der Grundlage eines djb-Seminars gaben die spätere Bundesverfassungsgerichtspräsidentin Prof. Dr. Jutta Limbach und die Universitätsprofessorin Dr. Ingeborg Schwenzer im Jahr 1988 das Buch „Familie ohne Ehe“ heraus. Teilweise ist es aber erst im 21. Jahrhundert zu vom Deutschen Juristinnenbund geforderten Angleichungen gekommen; manche lassen bis heute auf sich warten.

\section{Andere Rechtsgebiete}

Der Verfassungsauftrag zur Gleichberechtigung von Frau und Mann erforderte auch auf vielen anderen Rechtsgebieten neue Gesetze. Deutsche Juristinnen setzten sich seit der Verkündung des Grundgesetzes vielseitig ein, damit Diskriminierungen durch einfache Gesetze beendet bzw. erforderlichenfalls Gesetze zur Durchsetzung einer Gleichbehandlung geschaffen wurden.

\section{Beamtenrecht}

1953 wurde maßgeblich durch Zutun der Vereinigung weiblicher Juristen und Volkswirte die so genannte Zölibatsklausel aus dem Bundesbeamtengesetz entfernt. Zuvor konnten Beamtinnen, wenn sie heirateten, aus dem Dienst entlassen werden, sofern ihre wirtschaftliche Versorgung gewährleistet war. Es war ihnen gänzlich verwehrt, in Teilzeit zu arbeiten oder Familienurlaub zu nehmen. Die Rechtsanwältin und Bundestagsabgeordnete Dr. Emmy Diemer-Nicolaus reichte 1966 einen Entwurf der FDP-Fraktion zur Änderung des Bundesbeamtengesetzes ein. Er war vom Juristinnenbund zusammen mit dem Akademikerinnenbund erarbeitet worden und sah für Beamtinnen mit Kindern sowohl die Möglichkeit von Teilzeitarbeit als auch einer Beurlaubung vor. 1967 gründete der Juristinnenbund eine Kommission zu den verfassungs- und beamtenrechtlichen Fragen der Teilzeit von Beamtinnen und Richterinnen, deren Vorsitz Dr. Lore Maria Peschel-Gutzeit übernahm. Die im Wesent- lichen von Dr. Erna Scheffler erarbeitete Stellungnahme der Kommission hatte maßgeblichen Anteil daran, dass der Deutsche Bundestag am 31. März 1969 das 6. Gesetz zur Änderung beamtenrechtlicher und besoldungsrechtlicher Vorschriften beschloss. Fortan konnten Beamtinnen und Richterinnen mit Kindern in Teilzeit arbeiten oder beurlaubt werden.

\section{Schon 1977 forderte Dr. Annelies Kohleiss die renten- rechtliche Anerkennung von Erziehungszeiten - Gesetz wurde dies erst im Jahr 1998 nach drei einschlägigen Entscheidungen des Bundesverfassungsgerichts und zwei Rentenreformen.}

\section{Rentenrecht}

Zu einem großen Teil der Expertise und dem Engagement von Dr. Annelies Kohleiss ist es zu verdanken, dass Frauen im Rentenrecht ab den späten siebziger Jahren mehr Rechte erhielten. Seit 1970 Senatspräsidentin am Landessozialgericht BadenWürttemberg und Vorsitzende eines Rentenversicherungssenats, sah Dr. Annelies Kohleiss die eigenständige Sicherung der Frau in der Rentenversicherung als eine Lebensaufgabe an. Sie leitete die 1978 gegründete djb-Rentenkommission von der ersten Stunde an. Hier ging es im Schwerpunkt um die Neufassung der Hinterbliebenenversorgung zur eigenständigen Versorgung beider Ehegatten im Alter sowie die Berücksichtigung von Erziehungszeiten in der Altersvorsorge. Der djb gab darüber hinaus in wichtigen Gerichtsverfahren zu Rentenansprüchen - wie beispielsweise das Verfahren BVerfGE 53, 257 aus dem Jahr 1980, in dem der Versorgungsausgleich als mit Art. 14 GG vereinbar befunden wurde - vor dem Bundesverfassungsgericht Stellungnahmen ab, die in den Entscheidungen wie auch in der Öffentlichkeit deutliche Beachtung fanden.

Schon 1977 forderte Dr. Annelies Kohleiss die rentenrechtliche Anerkennung von Erziehungszeiten - Gesetz wurde dies erst im Jahr 1998 nach drei einschlägigen Entscheidungen des Bundesverfassungsgerichts und zwei Rentenreformen. Die Stellungnahmen von Dr. Annelies Kohleiss in rentenrechtlichen Fragen berücksichtigte das Bundesverfassungsgericht häufig. Im Verfahren BVerfGE 57, 335 etwa bestätigte es ihre Auffassung, indem es unterschiedliche Tabellenwerte für Ausbildungszeiten und die ersten fünf Versicherungsjahre von Männern und Frauen für verfassungswidrig erklärte (1981). Das vorgezogene Altersruhegeld für Frauen hielt das Bundesverfassungsgericht im Januar 1987 entsprechend der Stellungnahme von Dr. Annelies Kohleiss für verfassungsgemäß. 


\section{Steuerrecht}

Die Angleichung der Rechte von Frauen im Steuerrecht war dem Juristinnenbund nahezu seit seiner Gründung ein Anliegen. Dr. Erna Scheffler hatte schon 1950 auf dem 38. Deutschen Juristentag die damals geltende Haushaltsbesteuerung angegriffen; die Vereinigung weiblicher Juristen und Volkswirte forderte 1954 eine Individualbesteuerung. 1957 erklärte das Bundesverfassungsgericht die Haushaltsbesteuerung für verfassungswidrig - eine Entscheidung, an der Dr. Erna Scheffler beteiligt war. Wie sie hielt auch Dr. Annemarie Mennel, langjährige Vorsitzende der djb-Steuerkommission, das Ehegattensplitting für verfassungswidrig. Entsprechend lauteten die Forderungen Mennels auf dem 50. Deutschen Juristentag 1974. Darüber hinaus verlangte sie die Abzugsfähigkeit der Kosten für Kinderbetreuung. Der Gesetzgeber folgte diesen Forderungen nicht. Auch das Bundesverfassungsgericht sah die Abzugsfähigkeit nicht als von Verfassungs wegen geboten an, billigte sie aber immerhin Alleinerziehenden im Jahr 1982 zu. Eine Verfassungswidrigkeit des Ehegattensplittings sah das Bundesverfassungsgericht nicht.

\section{Strafrecht}

Im Strafrecht waren in der Zeit zwischen 1945 und 1989 insbesondere die Strafbarkeit des Schwangerschaftsabbruchs und die Reform des Sexualstrafrechts für die Arbeit des Deutschen Juristinnenbundes von Bedeutung. 1971 legte die Strafrechtskommission des Juristinnenbunds unter dem Vorsitz der Bundesrichterin Dr. Anne-Gudrun Meier-Scherling einen Regelungsvorschlag zur Straflosigkeit des Schwangerschaftsabbruchs innerhalb bestimmter Fristen oder bei bestimmten Indikationen vor. Das 5. Gesetz zur Reform des Strafrechts vom 18. Juni 1974 beinhaltete eine Fristenlösung, wurde aber vom Bundesverfassungsgericht als nicht verfassungskonform angesehen; eine Anpassung an die Vorgaben des Gerichts erfolgte durch das 15. Strafrechtsänderungsgesetz vom 18. Mai 1976. Ein Entwurf des Bundesfamilienministeriums aus dem Jahr 1988 bedingte eine erneute Auseinandersetzung des djb mit der Problematik. Er lehnte den Entwurf wegen seiner Unübersichtlichkeit und der ihm immanenten Bevormundung der Frau ab.

Im Sexualstrafrecht lag ein Schwerpunkt darauf, auch die Vergewaltigung in der Ehe zu sanktionieren. Die Strafrechtskommission des djb unter Leitung von der Prof. Dr. Monika Frommel legte 1988 den Vorschlag eines Gesetzes vor, der jedoch wie verschiedene andere parlamentarische Vorstöße zunächst ohne Konsequenzen blieb.

\section{Weitere}

Unter dem Vorsitz von Dr. Annelies Kohleiss näherten sich die Juristinnen des djb Anfang der 1980er Jahre darüber hinaus den Themen Gentechnik und Reproduktionstechnik. 1983 wurde unter der neuen Vorsitzenden Renate Damm eine Arbeitsgruppe zur Humangenetik und künstlichen Fortpflanzung gebildet.

Ende der 80er Jahre schließlich rückten Fragen der Gleichstellung der Frau im Erwerbsleben in den Vordergrund; dem- entsprechend war „Rechtsfragen der Gleichstellung der Frau im Erwerbsleben und Gesellschaft/Quotierungsfragen " Thema der 27. djb-Arbeitstagung 1987 in Hannover. Unter der Leitung von Juliane Freifrau von Friesen erarbeitete die neu gegründete djb-Kommission „Gleichberechtigung und Gleichstellung von Frauen und Männern in Beruf, Politik und Gesellschaft“ zur Mitgliederversammlung 1989 einen Bericht, der eklatante Mängel in der faktischen Gleichstellung offenbarte. Zur Herstellung eines verfassungskonformen Zustands wurde die Einführung von geschlechtsspezifischen Quoten empfohlen.

\section{Netzwerk}

Der djb verstand sich während der vielen Jahre seines Bestehens nicht nur als Wächter der Rechte von Frauen und Kindern, sondern leistete auch Unterstützung bei der Suche nach dem angemessenen Arbeitsplatz. Nach zahlreichen Anfragen junger Mitglieder gründete Hildegard Becker-Toussaint aus Frankfurt einen Arbeitsstab Karriereplanung, an dem sich nahezu alle Bundesvorstandsmitglieder beteiligten und ihre eigenen Erfahrungen weitergaben. Diese waren und sind noch immer vielfältig, hatten doch die „djb-Damen“ so ziemlich alle Chefetagen erklommen, die Juristinnen zugänglich waren, von den Bundesgerichten (ich denke nur an Antje SedemundTreiber und Elisabeth Steup als Präsidentinnen des Bundespatentgerichts, Marion Eckertz-Höfer als Präsidentin des Bundesverwaltungsgerichts, Dr. Iris Ebling als Präsidentin des Bundesfinanzhofs, Prof. Dr. Jutta Limbach als Präsidentin des Bundesverfassungsgerichts, die weiteren Verfassungsrichterinnen Prof. Dr. Karin Grasshof, Dr. Christine HohmannDennhardt, Dr. Gisela Niemeyer, Prof. Dr. Lerke Osterloh und Helga Seibert und an die Generalbundesanwältin Monika Harms) über die europäischen Gerichte (Dr. h.c. Renate Jaeger als deutsche Richterin beim Europäischen Gerichtshof für Menschenrechte und vormalige Richterin des BVerfG, Generalanwältin Prof. Dr. Juliane Kokott und Richterin Prof. Dr. Ninon Colneric am Europäischen Gerichtshof) und die Spitzenmedien (Renate Damm und Antje Pieper) bis zur Bundesregierung (Sabine Leutheusser-Schnarrenberger, Prof. Dr. Herta Däubler-Gmelin und Brigitte Zypries als Bundesministerinnen), dem Bundestag (Anke Fuchs als Bundestagsvizepräsidentin) und der Wissenschaft (Prof. Dr. Ursula Nelles als Rektorin der Universität Münster und die nicht mehr zu zählenden Wissenschaftlerinnen, die den besonderen fachlichen Ruf des djb verkörpern).

Nach der Wiedergründung des djb 1948 hätte sich niemand träumen lassen, dass man 60 Jahre später die Anzahl der erfolgreichen Richterinnen, Staatsanwältinnen und Rechtsanwältinnen nicht mehr auf einer Seite unterbringen kann, weil sich die Mitglieder des djb so zahlreich in allen juristischen und verwandten Bereichen durchgesetzt hatten. Das lag natürlich nicht nur am Arbeitsstab Karriereplanung, aber vielleicht auch an den vielen erfolgreichen Vorbildern im Verband.

Gerade Studentinnen und Juristinnen am Beginn ihrer Berufslaufbahn kann man deshalb nur raten, Mitglied zu werden. 